\title{
Seebeck coefficient in multiphase thin films
}

\author{
Nouredine Oueldna ${ }^{1,2}$, Alain Portavoce ${ }^{1, *}$, Maxime Bertoglio ${ }^{1}$, Marion Descoins ${ }^{1}$, \\ Abdelkhalek Kammouni ${ }^{2}$, and Khalid Hoummada ${ }^{1}$ \\ ${ }^{1}$ IM2NP, CNRS/Aix-Marseille University, Faculté des Sciences de Saint-Jérôme case 142, 13397 Marseille, \\ France \\ ${ }^{2}$ LASMAR, University of Moulay Ismail, Faculté des Sciences 11201 Meknes, Maroc
}

\begin{abstract}
$\mathrm{Mg}-\mathrm{Ag}-\mathrm{Sb}$ thin films were deposited by magnetron sputtering using a commercial $\mathrm{Mg}_{1 / 3} \mathrm{Ag}_{1 / 3} \mathrm{Sb}_{1 / 3}$ target. All the films contain principally the two phases $\mathrm{Ag}_{3} \mathrm{Sb}$ and $\alpha$-MgAgSb in same proportions but exhibit different microstructures. The films have the same Seebeck coefficient despite the difference of interface density and structure. Theoretical calculations show that the film effective Seebeck coefficient $S_{\text {film }}$ is depending only on the volume of each phase present in the film and their Seebeck coefficients. The multiple interfaces between the different phases have no effect on $S_{\text {film }}$.
\end{abstract}

Keywords: Thermoelectricity, Seebeck coefficient, Interface, Thin film, MgAgSb

*Corresponding author: alain.portavoce@im2np.fr 
Several solutions allowing the production of efficient thermoelectric materials were recently proposed [1]. Among them, charge carrier energy filtering is considered as one of the most promising strategy [1,2]. The principal method consists in the creation of specific interfaces in the material acting as energy filtering barriers, promoting a significant increase of the Seebeck coefficient $(S)$ and of the thermoelectric power factor. Experimental variations of $S$ in materials containing inclusions are regularly attributed to energy filtering. However, when several phases are present in a thermoelectric polycrystalline material, the effective Seebeck coefficient $S_{\text {eff }}$ is depending on $S_{\varphi i}$ of each present phase $\varphi_{i}$. Assuming a constant temperature $(T)$ gradient in the film, and excluding any interface effects, $S_{\text {eff }}$ of a material containing $n$ different phases can be expressed such as:

$$
S_{e f f}=\sum_{i}^{n} f_{\varphi_{i}} \times S_{\varphi_{i}}
$$

with $f_{\varphi}$ the volume fraction of the phase $\varphi_{i}$ (Fig. 1). In the present work, we investigate the interface influence on the $S$ of a polycrystalline material containing several phases. $\alpha$ $\mathrm{MgAgSb}$ is a ternary semi-conductor [3], which usually contains inclusions of the metallic binary phase $\mathrm{Ag}_{3} \mathrm{Sb}$ [3]. The $S$ of $\alpha-\mathrm{MgAgSb}$ was reported to significantly vary depending on the fabrication process [4-6]. $\alpha$-MgAgSb has been to date only studied in its bulk state, but $\alpha$ $\mathrm{MgAgSb}$ thin films produced by sputtering are expected to be useful for the integration of energy harvesting devices in the CMOS technology [7].

$\mathrm{Mg}-\mathrm{Ag}-\mathrm{Sb}$ thin films were deposited in a magnetron sputtering system exhibiting a base pressure of $10^{-8}$ Torr, using a $99.9999 \%$ pure Ar gas flow to sputter in the DC mode a 99.99\% pure $\mathrm{Mg}_{1 / 3} \mathrm{Ag}_{1 / 3} \mathrm{Sb}_{1 / 3}$ target purchased from CODEX international. The films were deposited on glass substrates for $S$ measurements, or on the native oxide of $\operatorname{Si}(001)$ substrates 
for electron microscopy observations. Four $300 \mathrm{~nm}$-thick $\mathrm{Mg}$-Ag-Sb films were deposited. Two films were deposited at room temperature (RT), the first one (\#1) was kept as-deposited, while the second one (\#2) was ex situ annealed under vacuum $\left(10^{-7}\right.$ mbar $)$ at $523 \mathrm{~K}$ for 30 min. The two other films were deposited at $523 \mathrm{~K}(\# 3)$ and $573 \mathrm{~K}(\# 4)$, respectively. The structure of the films was investigated using X-ray diffraction (XRD) in the Bragg-Brentano $(\theta-2 \theta)$ geometry using a $\mathrm{Cu} K_{\alpha}$ source $\left(\lambda_{K \alpha}=0.154 \mathrm{~nm}\right)$, as well as using scanning electron microscopy (SEM) in cross-section. The $S$ of the films was measured using a home-made setup. $T$ and the electrical potential were measured with the same probes at two different locations on the specimens separated by $1 \mathrm{~cm} . S$ was measured between $T=220$ and $330 \mathrm{~K}$, corresponding to the temperature range of the targeted CMOS applications. The experimental $S$ were compared to theory, running the BoltzTraP code [8] with material bulk parameters determined from ab-initio calculations. Ab-initio calculations were carried out using the firstprinciples pseudopotential method based on the DFT and the plane-wave self-consistent field method (PWSCF) which is implemented in the QUANTUM ESPRESSO package [9]. The Generalized Gradient Approximation (GGA-PBE) [10] was used for exchange-correlation functional. The ultrasoft pseudopotential (USP) method was used to treat the valence electron configuration.

Figure 2 presents the X-ray diffractograms measured on the four specimens. Fig. 2a shows that only the binary phase $\mathrm{Ag}_{3} \mathrm{Sb}$ is detected in the film after deposition at RT. Seven diffraction peaks are detected corresponding to the $\mathrm{Ag}_{3} \mathrm{Sb}$ atomic planes (110), (002), (111), (022), (130), (023), and (132) at $2 \theta=34.32^{\circ}, 37.24^{\circ}, 39.23^{\circ}, 51.56^{\circ}, 61.77^{\circ}, 68.44^{\circ}$, and $74.29^{\circ}$, respectively. Fig. $2 \mathrm{~b}$ shows that annealing the same film at $523 \mathrm{~K}$ for $30 \mathrm{~min}$ allows the phase $\alpha-\mathrm{MgAgSb}$ as well as the phase $\mathrm{Sb}$ to form in the film in addition to the phase $\mathrm{Ag}_{3} \mathrm{Sb}$. In addition to the same $\mathrm{Ag}_{3} \mathrm{Sb}$ diffraction peaks, five diffraction peaks corresponding 
to $\quad \alpha-\operatorname{MgAgSb}(110), \quad \alpha-\operatorname{MgAgSb}(202), \quad \alpha-\operatorname{MgAgSb}(114), \quad \alpha-\operatorname{MgAgSb}(330), \quad$ and $\quad \alpha-$ $\mathrm{MgAgSb}(006)$ are respectively observed in the diffractogram at $2 \theta=13.01^{\circ}, 24.16^{\circ}, 31.88^{\circ}$, $41.25^{\circ}$, and $42.06^{\circ}$, as well as two peaks corresponding to $\mathrm{Sb}(102)$ and $\mathrm{Sb}(213)$ at $2 \theta=$ $28.69^{\circ}$ and $48.35^{\circ}$. Fig. $2 \mathrm{c}$ and Fig. 2 d show that the same diffraction peaks are observed in the samples heated at $523 \mathrm{~K}$ or $573 \mathrm{~K}$ during deposition. Only the peak $\mathrm{Ag}_{3} \mathrm{Sb}$ (130) is only observed in the specimen ex situ annealed. The (qualitative) volume fraction $f$ of each phase present in the different films was determined using the integration of each detected peak of each phase. Due to the uncertainties related to the Bragg-Brentano geometry, the values of $f$ should not be considered quantitatively. Nevertheless, the fractions corresponding to the three phases present in the films are the same in the three different films \#2,\#3, and \#4: $f_{\alpha} \sim 33 \%$, $f_{A g 3 S b} \sim 60 \%$, and $f_{S b} \sim 7 \%$. Fig. 3 presents cross-sectional SEM images obtained on the films \#1 (Fig. 3a), \#2 (Fig. 3b), \#3 (Fig. 3c), and \#4 (Fig. 3d). The SEM images show in the four films the coexistence of multiple grains of different phases exhibiting different contrasts. However, the microstructures of the samples \#2, \#3, and \#4 are different. According to the $\mathrm{XRD}$ analyses, the matrix should correspond to the phase $\mathrm{Ag}_{3} \mathrm{Sb}$, while the inclusions should be $\alpha-\mathrm{MgAgSb}$ grains. The inclusions in the film \#2 exhibit a higher density $\left(\rho \sim 1.5 \times 10^{11}\right.$ $\left.\mathrm{cm}^{-2}\right)$ and smaller sizes $(10 \leq l \leq 50 \mathrm{~nm})$ than in the film \#3 $\left(\rho \sim 8.2 \times 10^{9} \mathrm{~cm}^{-2}, 23 \leq l \leq\right.$ $70 \mathrm{~nm})$ and \#4 $\left(\rho \sim 3.5 \times 10^{9} \mathrm{~cm}^{-2}, 65 \leq l \leq 90 \mathrm{~nm}\right)$. Furthermore, the film \#3 is rougher and facets are less easily observed on the inclusions compared to film \#4. Fig. 4 presents $S$ measured on the four different films versus T. $S$ measured on the films \#2 (open up triangles), \#3 (solid circles), and \#4 (solid squares), are similar in the considered $T$ range. However, $S$ measured on the as-deposited film \#1 (solid down triangles) is significantly lower than the three other films. Indeed, $S$ measured in the film \#1 should correspond to the phase $\mathrm{Ag}_{3} \mathrm{Sb}$, while $S$ measured on the three other films should correspond to an effective coefficient resulting from the presence of the different phases. Fig. 4 shows also the theoretical $S$ of the 
two major phases present in the samples (i.e. $\mathrm{Ag}_{3} \mathrm{Sb}$ and $\alpha$-MgAgSb) calculated using the BoltzTraP code and ab-initio calculations. The theoretical coefficient of $\mathrm{Ag}_{3} \mathrm{Sb}$ (solid line) is in good agreement with the experimental coefficient measured on the film \#1 (solid down triangles), containing only $\mathrm{Ag}_{3} \mathrm{Sb}$. Furthermore, the theoretical coefficient of $\alpha-\mathrm{MgAgSb}$ is in good agreement with the experimental value reported by Kirkham et al. [4] (solid diamonds). XRD measurements suggest that the phase $\alpha$-MgAgSb occupies in average only $30 \%$ of the volume of the films \#2, \#3, and \#4. Neglecting the presence of Sb in the samples, $S_{\text {eff }}$ of a hypothetic film containing the two phases $\mathrm{Ag}_{3} \mathrm{Sb}$ and $\alpha$-MgAgSb was calculated using the theoretical values of $S_{\mathrm{Ag} 3 \mathrm{Sb}}$ and $S_{\alpha}$ and eq. 1 , with $f_{\alpha}=0.3$ and $f_{\text {Ag3Sb }}=0.7 . S_{\text {eff }}$ (dash line in Fig. 4) is in good agreement with the $S$ measured on the three films \#2, \#3, and \#4. Neither nano-size effect [11-12] nor interfacial effect [1-2] are observed on $S$. Indeed, the microstructures of the films may be too far from the structure of superlattice-type materials, the size of $\alpha-\mathrm{MgAgSb}$ inclusions being too large (> $5 \mathrm{~nm}$ [11-12]) and exhibiting a significant size distribution. Furthermore, the nature of the $\mathrm{Ag}_{3} \mathrm{Sb} / \alpha-\mathrm{MgAgSb}$ interface may not promote the suitable energy barrier (ohmic instead of Schottky contact).

In conclusion, $\mathrm{Mg}-\mathrm{Ag}-\mathrm{Sb}$ thin films were elaborated by magnetron sputtering in different conditions allowing the production of different films containing principally the phase $\mathrm{Ag}_{3} \mathrm{Sb}$ and the phase $\alpha-\mathrm{MgAgSb}$ in same proportions. These films exhibit the same $S_{\text {eff }}$ despite a significant difference of interface density and structure. $S_{\text {eff }}$ can be determined considering the Seebeck coefficients of the two different phases and their volume in the films, without considering any influence of the interfaces.

This work was supported by the project PHC Toubkal TBK/18/65 - Campus France $\mathrm{N}^{\circ}$ 39048SE. 


\section{References}

[1] J. He, T.M. Tritt, Science. 357 (2017) 1369.

[2] D. Narducci, S. Frabboni, X. Zianni, J. Mater. Chem. C. 3 (2015) 12176.

[3] Z. Liu, J. Mao, J. Sui, Z. Ren, Energy Environ. Sci. (2017) 0.

[4] M.J. Kirkham, A.M. dos Santos, C.J. Rawn, E. Lara-Curzio, J.W. Sharp, A.J. Thompson, Phys. Rev. B - Condens. Matter Mater. Phys. 85 (2012) 144120.

[5] P. Ying, X. Liu, C. Fu, X. Yue, H. Xie, X. Zhao, W. Zhang, T. Zhu, Chem. Mater. 27 (2015) 909.

[6] H. Zhao, J. Sui, Z. Tang, Y. Lan, Q. Jie, D. Kraemer, K. McEnaney, A. Guloy, G. Chen, Z. Ren, Nano Energy. 7 (2014) 97.

[7] J.A. Kittl, K. Opsomer, C. Torregiani, C. Demeurisse, S. Mertens, D.P. Brunco, M.J.H. Van Dal, A. Lauwers, Mater. Sci. Eng. B Solid-State Mater. Adv. Technol. 154 (2008) 144.

[8] G.K.H. Madsen, D.J. Singh, Comput. Phys. Commun. 175 (2006) 67.

[9] P. Giannozzi, S. Baroni, N. Bonini, et al, J. Phys. Condens. Matter. 21 (2009).

[10] J.P. Perdew, K. Burke, M. Ernzerhof, Journal of Physics C: Solid State Physics, 12(22), 4977., Phys. Rev. Lett. 77 (1996) 3865.

[11] V. Vargiamidis, M. Thesberg, and N. Neophytou, J. Appl. Phys. 126 (2019) 055105.

[12] N. Neophytou, S. Foster, V. Vargiamidis, G. Pennelli, D. Narducci, Mat. Today Phys., https://doi.org/10.1016/j.mtphys.2019.100159 


\section{CAPTIONS}

FIG. 1. Schematic showing the serial electrical circuit corresponding to the case of a thermoelectric material made of two different phases $\varphi 1$ and $\varphi 2$ in a temperature gradient, $S_{\varphi 1}$ (resp. $S_{\varphi 2}$ ) and $\sigma_{\varphi 1}$ (resp. $\sigma_{\varphi 2}$ ) being the Seebeck coefficient and the electrical conductivity of the phase $\varphi 1$ (resp. $\varphi 2$ ). Any $\varphi 1 / \varphi 2$ interface effect is neglected.

FIG. 2. Ex situ X-ray diffractograms acquired in the $\theta-2 \theta$ geometry on the four different films: \#1 (a), \#2 (b), \#3 (c), and \#4 (d).

FIG. 3. Cross-sectional SEM images acquired on the four different films: a) RT as-deposited film (film \#1), b) RT deposited film annealed under vacuum at $523 \mathrm{~K}$ for $30 \mathrm{~min}$ (film \#2), c) film deposited at $523 \mathrm{~K}$ (film \#3), and d) film deposited at $573 \mathrm{~K}$ (film \#4).

FIG. 4. Comparison between experimental (solid symbols) and calculated (lines) Seebeck coefficients $(S)$ for $220 \leq T \leq 330 \mathrm{~K}$ : The solid down triangles, the solid circles, and the solid squares correspond to the RT as-deposited film (film \#1), the film deposited at $523 \mathrm{~K}$ (film \#3), and the film deposited at $573 \mathrm{~K}$ (film \#4). The open up triangles corresponds to the RT deposited film annealed under vacuum at $523 \mathrm{~K}$ for $30 \mathrm{~min}$ (film \#2) and the solid diamonds corresponds to the phase $\alpha-\mathrm{MgAgSb}$ from [4]. The lines correspond to the $S$ theoretical values of bulk $\mathrm{Ag}_{3} \mathrm{Sb}$ (solid line), bulk $\alpha-\mathrm{MgAgSb}$ (dash dot line), and a film containing in its volume $70 \%$ of $\mathrm{Ag}_{3} \mathrm{Sb}$ and $30 \%$ of $\alpha-\mathrm{MgAgSb}$, neglecting $\mathrm{Ag}_{3} \mathrm{Sb} / \alpha-\mathrm{MgAgSb}$ interface effects (dash line). 
Figure 1

N. Oueldna et al.

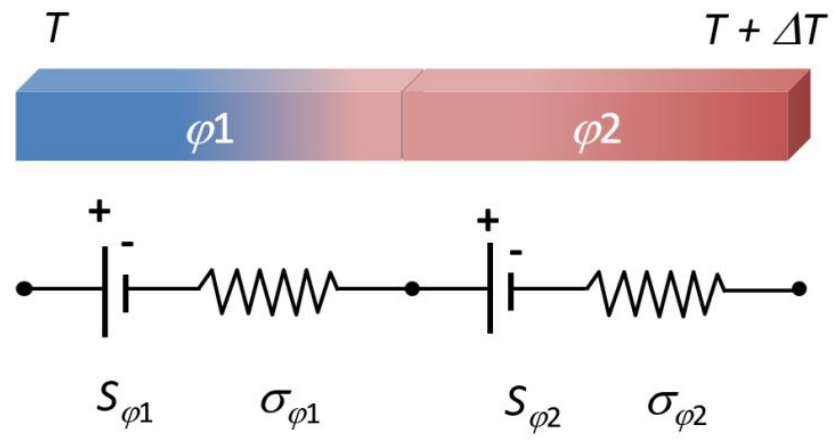


Figure 2

N. Oueldna et al.

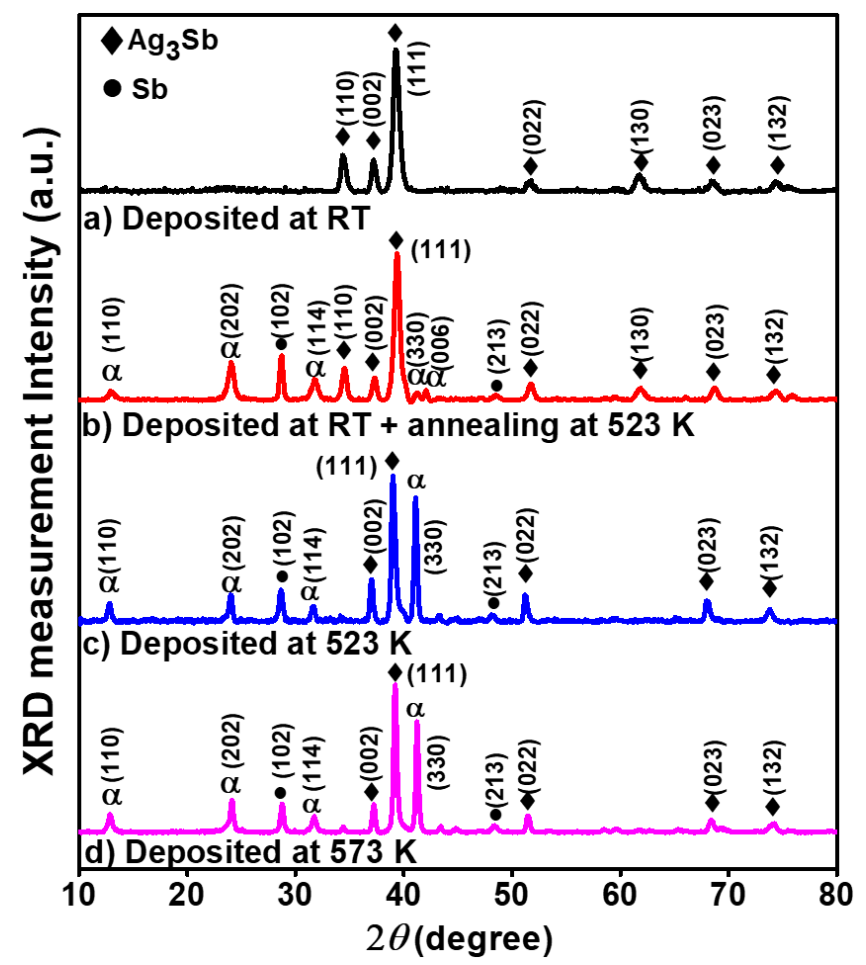




\section{Figure 3}

N. Oueldna et al.

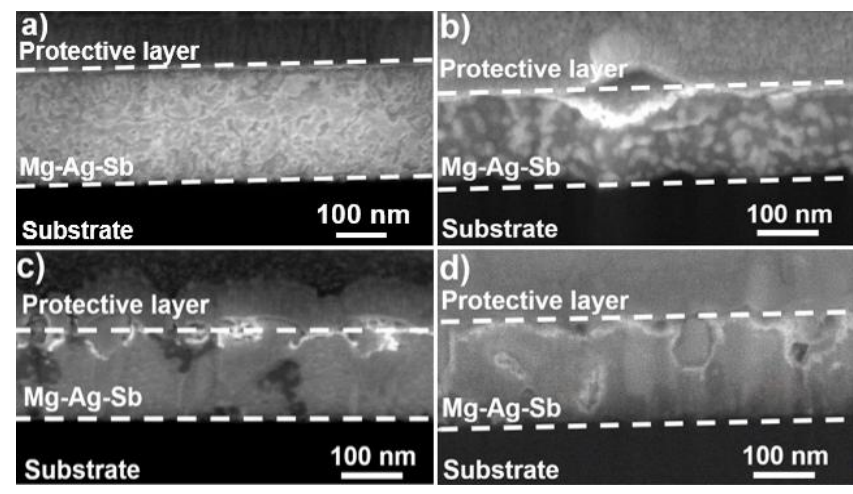


Figure 4

N. Oueldna et al.

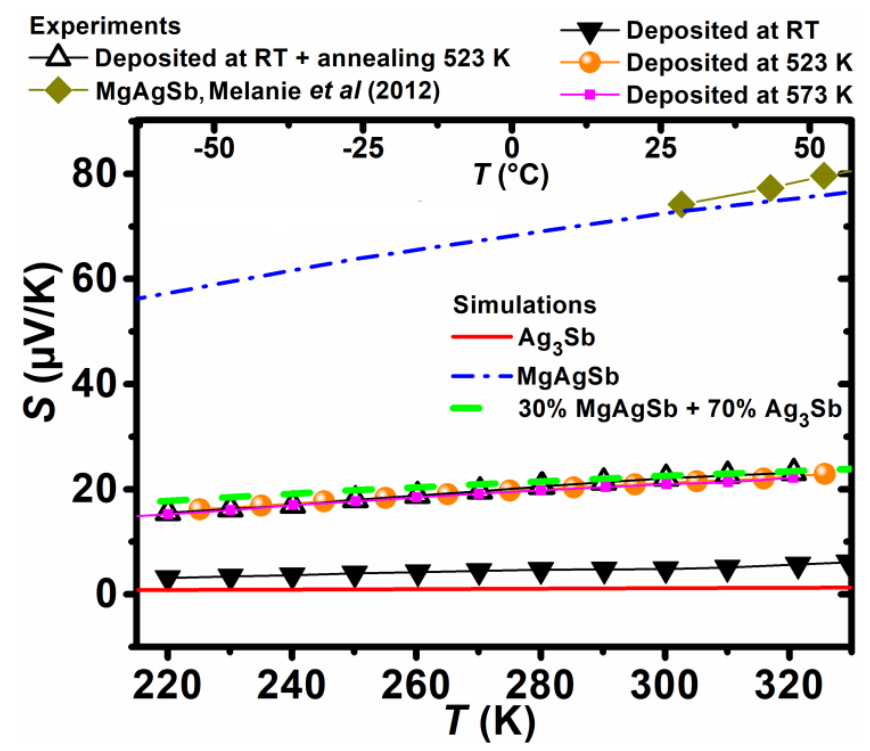

